\title{
The Association Between Serum Anion Gap and All-Cause Mortality in Disseminated Intravascular Coagulation Patients: A Retrospective Analysis
}

\author{
Bin $\mathrm{Hu}^{\prime}$ \\ Jinxia Cao' \\ Yangyang $\mathrm{Hu}^{2}$ \\ Zuoan Qin $^{3}$ \\ Jun Wang'
}

'Department of Hematology, First People's Hospital of Changde City, Changde, Hunan, People's Republic of China; ${ }^{2}$ Department of Rheumatology and Immunology, Tongji Hospital, Tongji Medical College, Huazhong University of Science and Technology, Wuhan, Hubei, People's Republic of China; ${ }^{3}$ Department of Cardiovascular Medicine, First People's Hospital of Changde City, Changde, Hunan, People's Republic of China
Correspondence: Jun Wang Department of Hematology, First People's Hospital of Changde City, 818 Renmin Road, Changde, 415000, Hunan, People's Republic of China

Tel +86 736-7788720

Email xueyeke2020@sina.com
Purpose: Studies regarding death risk factors of disseminated intravascular coagulation (DIC) patients were limited. Therefore, we conducted this study to investigate whether the serum anion gap (AG) was independently related to all-cause mortality of DIC patients.

Methods: We used the data from Medical Information Mart for Intensive Care III version 1.4 (MIMIC-III v1.4). A total of 2,654 DIC patients were included. The main outcomes were in-hospital, 30-day, and 90-day all-cause mortality. The AG was measured upon ICU admission and its association with mortality was evaluated using the Cox proportionalhazards regression model. The generalized additive model and the smooth curve fitting were introduced to examine the non-linear association.

Results: After adjusting for potential covariates, the in-hospital, 30-day, and 90-day allcause mortality were positively correlated with AG. The hazard ratio (HR), confidence intervals $(\mathrm{CI})$, and $P$ were $1.05(1.04-1.07)<0.0001,1.06(1.04-1.07)<0.0001$, and 1.05 $(1.03-1.07)<0.0001$, respectively. We did not find an obvious non-linear relationship between AG and in-hospital, 30-day, and 90-day mortality, which indicated that the association between AG and all-cause mortality of DIC patients was nearly linear.

Conclusion: Serum AG is positively related with all-cause mortality in DIC patients.

Keywords: anion gap, disseminated intravascular coagulation, all-cause mortality, MIMICIII, retrospective study

\section{Introduction}

Disseminated intravascular coagulation (DIC) is a clinically severe syndrome characterized by uncontrolled and widespread activation of blood coagulation, which is caused by serious infections, trauma, malignancies, liver diseases, obstetric diseases, etc. ${ }^{1}$ The pathophysiological mechanisms of DIC includes activation of tissue factor-related intravascular coagulation, a dysfunction of anticoagulant pathways and suppression of fibrinolysis caused by plasminogen activator inhibitor. ${ }^{2}$ Together, these changes may result in micro-vascular thrombosis, multiple organ dysfunction, and severe bleeding tendency, which always lead to a significantly increased mortality. ${ }^{2,3}$ Mortality risks have been demonstrated to double in critically ill patients if DIC occurred. ${ }^{4}$ Because of the poor prognosis of DIC, it is necessary to identify its potential death risk factors. However, there are currently few clinical studies focusing on this field.

The development of DIC is closely related to acid-base imbalance. ${ }^{5,6}$ Acid-base imbalance is considered as one of the initiating factors resulting in DIC., ${ }^{5,6}$ Severe 
acidosis may injure the endothelial cell, which may further lead to the tissue factor release. ${ }^{7}$ The tissue factor-factor VII pathway is the principal initiator of thrombin generation in DIC. ${ }^{2}$ Moreover, DIC is often associated with micro-vascular thrombosis and micro-circulatory dysfunction, which may further lead to or worsen metabolic acidosis. ${ }^{8}$ Metabolic acidosis is common in DIC patients and it may be a strong predictor of its prognosis.

The serum anion gap (AG), calculated by the formula $\left(\mathrm{Na}^{+}+\mathrm{K}^{+}\right)-\left(\mathrm{Cl}^{-}+\mathrm{HCO}^{-}\right)$, reflects the concentration of unmeasured serum anions. ${ }^{9,10}$ AG is widely used in analysis of acid-base disorders and high values of AG always indicate metabolic acidosis. ${ }^{10}$ The level of serum AG is closely related to the severity of metabolic acidosis. ${ }^{11}$

On the basis of the close pathophysiological relationship among DIC, metabolic acidosis, and AG, we speculate that AG might be one of the prognostic predictors of DIC patients. Furthermore, previous clinical research has demonstrated that high AG was an independent adverse prognostic factor in a variety of diseases, ${ }^{12}$ such as coronary artery disease, ${ }^{13}$ acute kidney injury, ${ }^{14}$ and aortic aneurysm. ${ }^{15} \mathrm{Up}$ to now, there is no clinical research focusing on the association between $\mathrm{AG}$ and DIC prognosis. We conducted this work to evaluate whether AG was independently related to all-cause mortality of DIC patients.

We used the data from the Medical Information Mart for Intensive Care III version 1.4 (MIMIC-III v1.4), which is a publicly available database enrolling 46,476 patients and 61,532 admissions to intensive care units (ICU). ${ }^{16}$ In total, 3,044 DIC patients were identified from MIMIC-III. We produced a series of clinical studies regarding mortality risk factors in DIC patients, and this paper is the first report of our studies.

\section{Methods}

\section{Database}

The MIMIC-III database is approved by the Massachusetts Institute of Technology and the Institutional Review Boards of Beth Israel Deaconess Medical Center. ${ }^{16}$ The authors, Bin $\mathrm{Hu}$ and Zuoan Qin, finished the Collaborative Institutional Training Initiative (CITI) program course named "Data or Specimens Only Research" and achieved access to the database (Record ID: 35942628 and 36208651).

\section{Data Extraction}

The Structure Query Language (SQL) with PostgreSQL (version 9.6) was introduced to extract the data from the
MIMIC-III database. The following information were collected: general information, causes of DIC, vital signs, scoring systems (sequential organ failure assessment $\left(\mathrm{SOFA}^{17}\right)$ score, systemic inflammatory response syndrome $\left(\operatorname{SIRS}^{18}\right)$ score, and simplified acute physiology score II $\left(\mathrm{SAPSII}^{19}\right)$ ), comorbidities (hypertension, diabetes, chronic obstructive pulmonary disease (COPD), liver diseases, renal failure), laboratory data (AG, hemoglobin, hematocrit, red blood cell distribution (RDW), platelet, white blood cell (WBC), activated partial thromboplastin time (APTT), prothrombin time (PT), international normalized ratio (INR), creatinine, urea nitrogen, glucose, potassium, sodium, bicarbonate, albumin, and lactate), and treatment (continuous renal replacement therapy (CRRT), vasopressor drugs and mechanical ventilation). It should be noted that all laboratory results we extracted were the first test results after admission to the ICU. The primary endpoint of this study was in-hospital, 30-day, and 90-day all-cause mortality from admission to the ICU.

\section{Inclusion Criteria}

The diagnosis of DIC was established using the scoring system recommended by the Japanese Association of Acute Medicine (JAAM). ${ }^{20}$ We calculated patients' JAAM-DIC scores according to their SIRS scores, platelet count, fibrin degradation products (FDP) and, prothrombin time- international normalized ratio (PT-INR) within 24 hours of admission to the ICU. Patients were diagnosed as DIC if their JAAM-DIC scores were $\geq 4 .{ }^{20}$ Exclusion criteria were as follows:

- non-first admission to ICU;

- age $<14$ or $>89$ years old;

- a length of ICU stay less than 24 hours; and

- missing AG data at ICU admission.

\section{Statistical Analysis}

To examine the link between AG and the risk of all-cause mortality of DIC patients, we introduced three different models by univariate and multivariate Cox proportionalhazards regression model, including non-adjusted model (no covariates were adjusted), minimally-adjusted model (only sociodemographic variables were adjusted), and fullyadjusted model (all potential covariates were adjusted). Effect sizes with $95 \%$ confidence intervals were recorded. Accounting for the non-linear correlation between $\mathrm{AG}$ and all-cause mortality of DIC patients, we also used a generalized additive model and the smooth curve fitting 


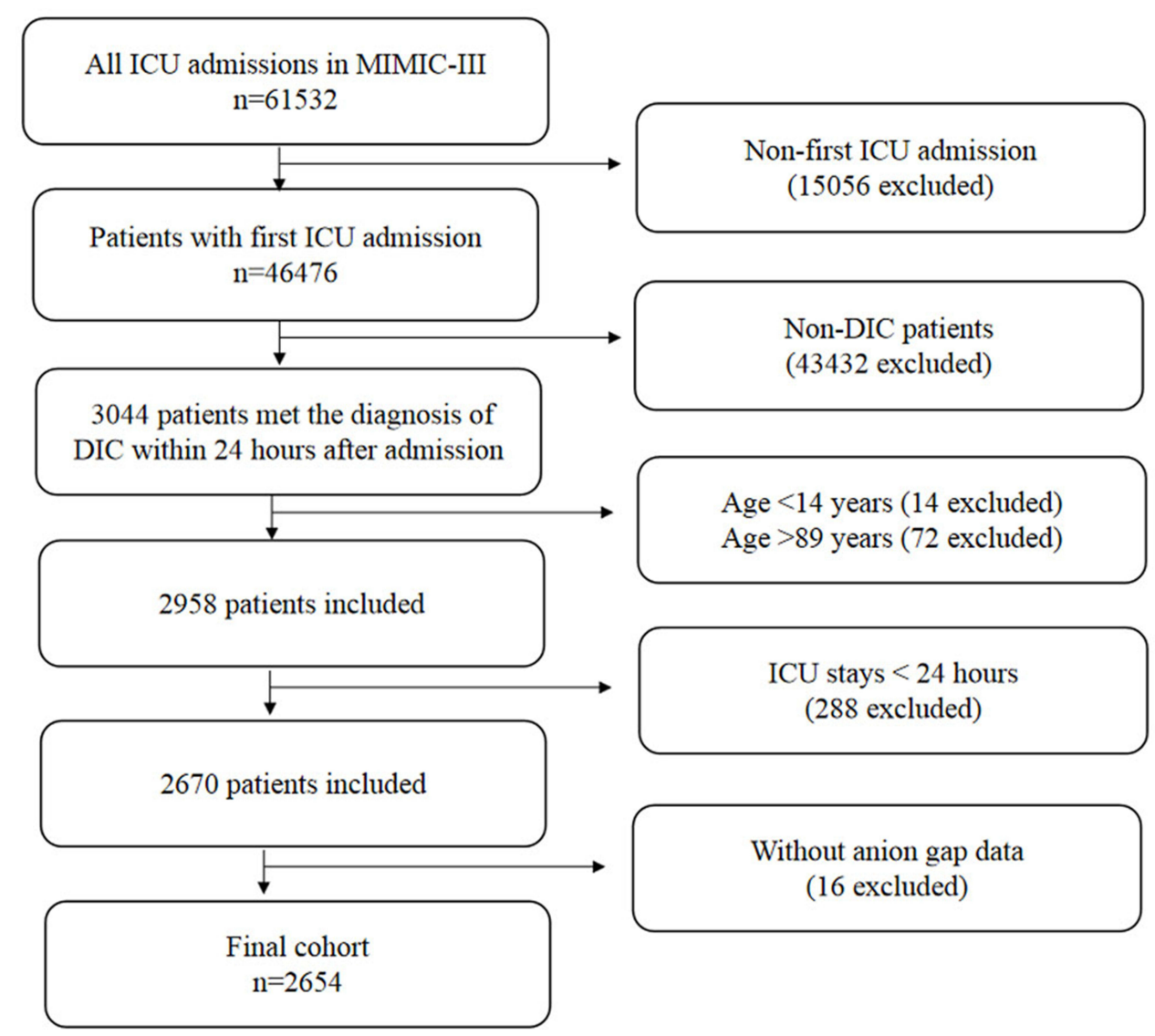

Figure I Flowchart of subject screening.

(penalized spline method) to address non-linearity. The subgroup analyses were conducted using a stratified Cox proportional-hazards regression model. To test the robustness of our results, we performed a sensitivity analysis. We converted $A G$ into a categorical variable according to the bisected, and calculated the $P$ for trend in order to verify the results of $A G$ as the continuous variable. Modeling was performed with the statistical software packages R (http:// www.R-project.org, The R Foundation) and EmpowerStats (http://www.empowerstats.com, X\&Y Solutions, Inc, Boston, MA). $P$-values less than 0.05 (two-sided) were considered statistically significant.

\section{Results}

\section{Patients Characteristics}

The detailed process of patients selection is shown in Figure 1. Initially, 46,476 patients with 61,532 admissions to the ICU were selected from the MIMIC-III database. In total, 3,044 patients fulfilled the diagnostic criteria of DIC. Of these, 2,654 patients fulfilled the inclusion criteria mentioned above and were enrolled in this study. Table 1 lists the baseline characteristics of the included patients. Their average age was $60.55 \pm 16.23$ years old and $59.2 \%$ were male. The 2,654 patients were divided into four groups using the quartiles of AG level. There were 642 patients in the 4.00 $11.00 \mathrm{mmol} / \mathrm{L}$ group, 540 patients in the $12.00-13.00 \mathrm{mmol} /$ $\mathrm{L}$ group, 787 patients in the $14.00-17.00 \mathrm{mmol} / \mathrm{L}$ group, and 685 patients in the $18.00-48.00 \mathrm{mmol} / \mathrm{L}$ group.

\section{The Association Between AG and All-Cause Mortality}

Both univariate and multivariate Cox proportional-hazards regression models were introduced to evaluate the associations between AG and in-hospital, 30-day, and 90-day mortality of DIC patients (Table 2). Meanwhile, we show the non-adjusted and adjusted models in Table 2. We found that higher AG was associated with increased risk of in-hospital mortality (non-adjusted model: $\mathrm{HR}=1.08,95 \% \mathrm{CI}=1.07-$ 1.10, $P<0.0001$; minimally-adjusted model: $\mathrm{HR}=1.09$, 95\% CI $=1.07-1.10, \quad P<0.0001$; fully-adjusted model: $\mathrm{HR}=1.05,95 \% \mathrm{CI}=1.04-1.07, P<0.0001)$, 30-day mortality (non-adjusted model: $\quad \mathrm{HR}=1.10, \quad 95 \% \quad \mathrm{CI}=1.08-1.11$, 


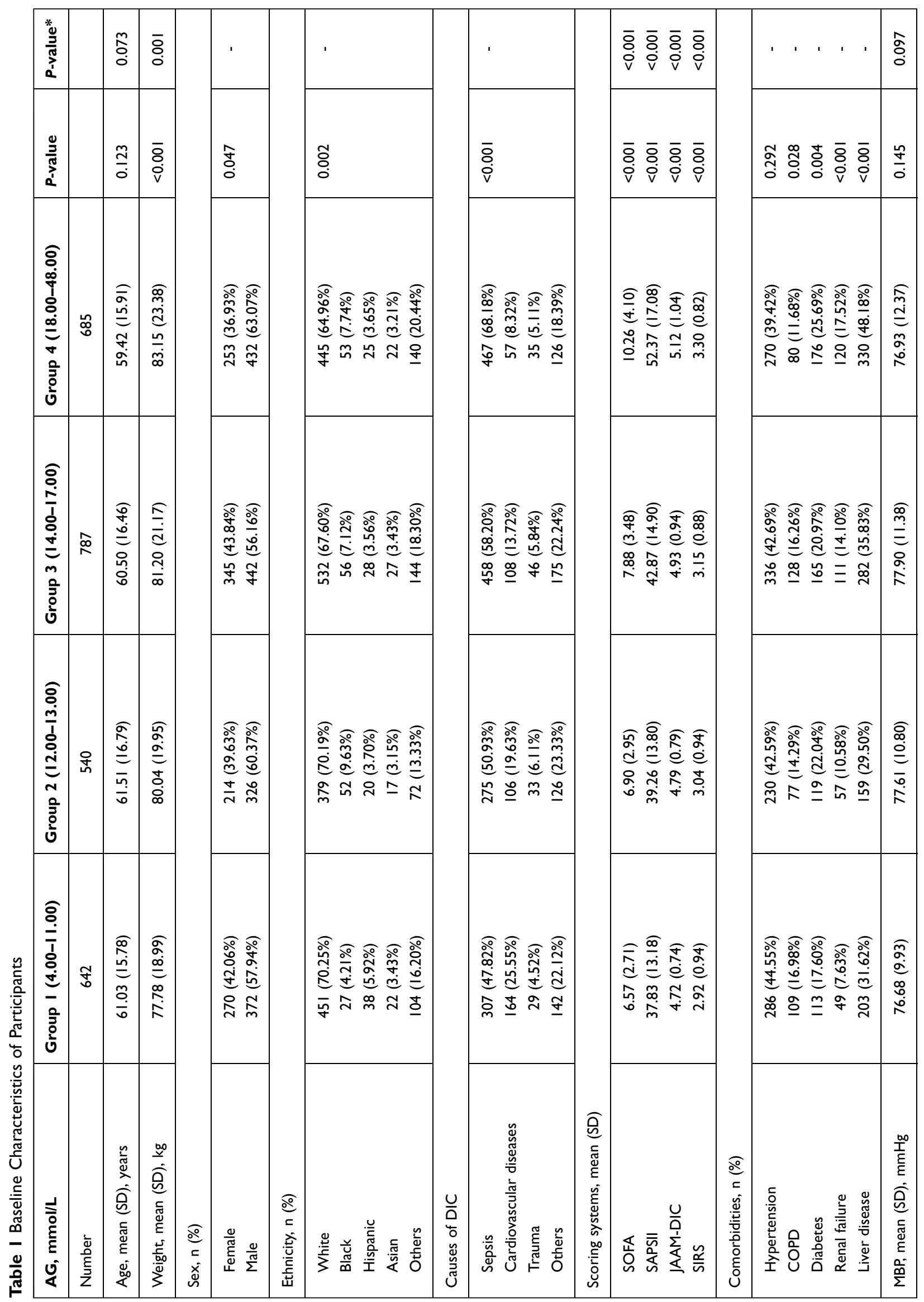




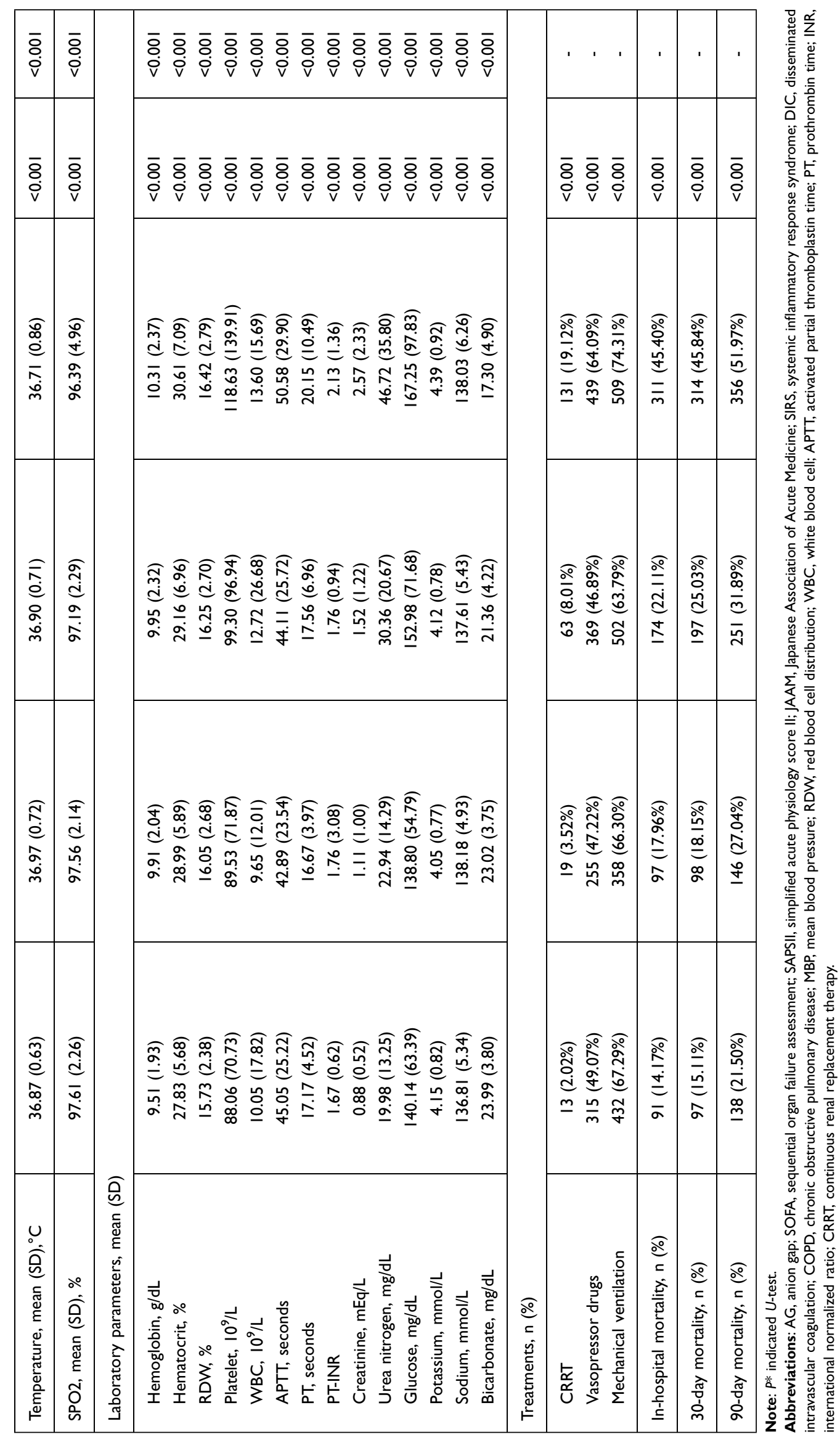


Table 2 Univariate and Multivariate Results by Cox Regression

\begin{tabular}{|c|c|c|c|}
\hline Exposure & $\begin{array}{l}\text { Non-Adjusted Model } \\
\text { HR, } 95 \% \mathrm{CI}, \mathrm{P}\end{array}$ & $\begin{array}{l}\text { Minimally-Adjusted Model } \\
\text { HR, } 95 \% \mathrm{Cl}, \mathrm{P}\end{array}$ & $\begin{array}{l}\text { Fully-Adjusted Model } \\
\text { HR, } 95 \% \mathrm{CI}, \text { P }\end{array}$ \\
\hline $\begin{array}{l}\text { In-hospital mortality } \\
\text { Anion gap }\end{array}$ & $1.08(1.07-1.10)<0.0001$ & $1.09(1.07-1.10)<0.0001$ & $1.05(1.04-1.07)<0.0001$ \\
\hline $\begin{array}{l}\text { Anion gap (quartiles) } \\
\text { Q1 } \\
\text { Q2 } \\
\text { Q3 } \\
\text { Q4 } \\
\text { P for trend }\end{array}$ & $\begin{array}{l}\text { I.0 (Ref) } \\
\text { I.I9 (0.90-I.59) } 0.2268 \\
1.32(1.02-1.70) 0.0336 \\
2.92(2.31-3.69)<0.000 \text { I } \\
\text { I.47 (I.37-I.59) <0.000। }\end{array}$ & $\begin{array}{l}\text { I.0 (Ref) } \\
\text { I.I7(0.88-I.56) } 0.2719 \\
I .32(I .02-I .70) 0.0340 \\
3.00(2.37-3.79)<0.000 \text { I } \\
I .49(I .38-I .6 I)<0.000 \text { I }\end{array}$ & $\begin{array}{l}\text { I.0 (Ref) } \\
\text { I.07 }(0.80-1.44) 0.6444 \\
\text { I.02 }(0.78-1.33) 0.8943 \\
\text { I.83 }(1.38-2.43)<0.000 \text { I } \\
\text { I.22 }(I . I I-1.34)<0.000 \text { I }\end{array}$ \\
\hline $\begin{array}{l}\text { 30-day mortality } \\
\text { Anion gap }\end{array}$ & $1.10(1.08-1.1 I)<0.000 I$ & $1.10(1.09-1.11)<0.0001$ & $1.06(1.04-1.07)<0.0001$ \\
\hline $\begin{array}{l}\text { Anion gap (quartiles) } \\
\text { Q1 } \\
\text { Q2 } \\
\text { Q3 } \\
\text { Q4 } \\
\text { P for trend }\end{array}$ & $\begin{array}{l}\text { I.0 (Ref) } \\
\text { I.22 }(0.92-1.62) 0.1586 \\
\text { I.74 (I.37-2.22)<0.000। } \\
3.92(3.12-4.92)<0.000 \text { I } \\
\text { I.64 }(I .52-1.77)<0.000 \text { I }\end{array}$ & $\begin{array}{l}\text { I.0 (Ref) } \\
\text { I.22 }(0.92-1.62) 0.1635 \\
\text { I.76 (I.38-2.24)<0.000। } \\
4.04(3.22-5.08)<0.000 \text { I } \\
\text { I.66 }(I .54-1.79)<0.000 \text { I }\end{array}$ & $\begin{array}{l}\text { I.0 (Ref) } \\
\text { I.06 (0.79-I.4I) } 0.6963 \\
\text { I.23 (0.95-I.59) } 0.1122 \\
\text { I. } 97(I .49-2.60)<0.000 \text { I } \\
\text { I. } 26(I .15-1.39)<0.000 \text { I }\end{array}$ \\
\hline $\begin{array}{l}\text { 90-day mortality } \\
\text { Anion gap }\end{array}$ & $1.09(1.08-1.10)<0.0001$ & $1.09(1.08-1.10)<0.0001$ & $1.05(1.03-1.07)<0.000$ I \\
\hline $\begin{array}{l}\text { Anion gap (quartiles) } \\
\text { Q1 } \\
\text { Q2 } \\
\text { Q3 } \\
\text { Q4 } \\
\text { P for trend }\end{array}$ & $\begin{array}{l}\text { I.0 (Ref) } \\
\text { I.29 }(1.02-1.63) 0.0306 \\
1.59(1.29-1.96)<0.000 \text { I } \\
3.27(2.69-3.99)<0.000 \text { I } \\
1.50(1.41-1.60)<0.000 \text { I }\end{array}$ & $\begin{array}{l}1.0 \text { (Ref) } \\
1.29(1.02-1.63) 0.0320 \\
1.61(1.31-1.98)<0.0001 \\
3.39(2.78-4.13)<0.0001 \\
1.52(1.43-1.62)<0.0001\end{array}$ & $\begin{array}{l}1.0 \text { (Ref) } \\
\text { I.I5 }(0.91-1.46) 0.2496 \\
\text { I.I5 }(0.92-1.43) 0.2170 \\
\text { I.73 }(1.35-2.20)<0.000 \text { I } \\
\text { I.I8 }(1.09-1.28)<0.000 \text { I }\end{array}$ \\
\hline
\end{tabular}

Notes: Non-adjusted model: no covariates were adjusted for. Minimally-adjusted model: we only adjusted for age and sex. Fully-adjusted model: we adjusted for age, sex, ethnicity, causes of DIC, SOFA score, SAPSII score, renal failure, CRRT, vasopressor drugs, mechanical ventilation, APTT, PT-INR, bicarbonate, creatinine, potassium, urea nitrogen, and RDW.

Abbreviations: $\mathrm{Cl}$, confidence interval; Ref, reference.

$P<0.0001$; minimally-adjusted model: $\mathrm{HR}=1.10,95 \%$ $\mathrm{CI}=1.09-1.11, P<0.0001$; fully-adjusted model: $\mathrm{HR}=1.06$, 95\% CI=1.04-1.07, $P<0.0001$ ), and 90-day mortality (nonadjusted model: $\mathrm{HR}=1.09,95 \% \mathrm{CI}=1.08-1.10, P<0.0001$; minimally-adjusted model: $\mathrm{HR}=1.09,95 \% \mathrm{CI}=1.08-1.10$, $P<0.0001$; fully-adjusted model: $\mathrm{HR}=1.05,95 \% \mathrm{CI}=1.03-$ $1.07, P<0.0001)$. For the purpose of sensitivity analysis, we also handled AG as a categorical variable (quartile), and found the same trend (Table 2).

\section{The Results of the Non-Linear Relationship}

We did not find an obvious non-linear relationship between AG and in-hospital mortality (Figure 2A), 30-day mortality (Figure 2B), or 90-day mortality (Figure 2C) using the generalized additive model and the smooth curve fitting. The association between AG and all-cause mortality of DIC patients was nearly linear.

\section{Subgroup Analyses}

Table 3 displays the results of subgroup analyses regarding the outcome of 30-day mortality. The tests for interactions were not statistically significant for sex, age, care unit, causes of DIC, COPD, diabetes, CRRT use, vasopressor drugs use, or mechanical ventilation use $(P$ for interaction $=0.7543$, $0.2294,0.4727,0.1526,0.3802,0.2487,0.7379,0.9986$, and 0.2795$)$. The test for interactions were significant for renal failure ( $P$ for interaction $=0.0310$ ). The positive association between AG and 30-day mortality was more obvious in DIC patients with renal failure $(\mathrm{HR}=1.10,95 \% \mathrm{CI}=1.06$ $1.14, P<0.0001)$ than those without renal failure $(\mathrm{HR}=1.05$, 95\% CI=1.03-1.07, $P<0.0001)$. 

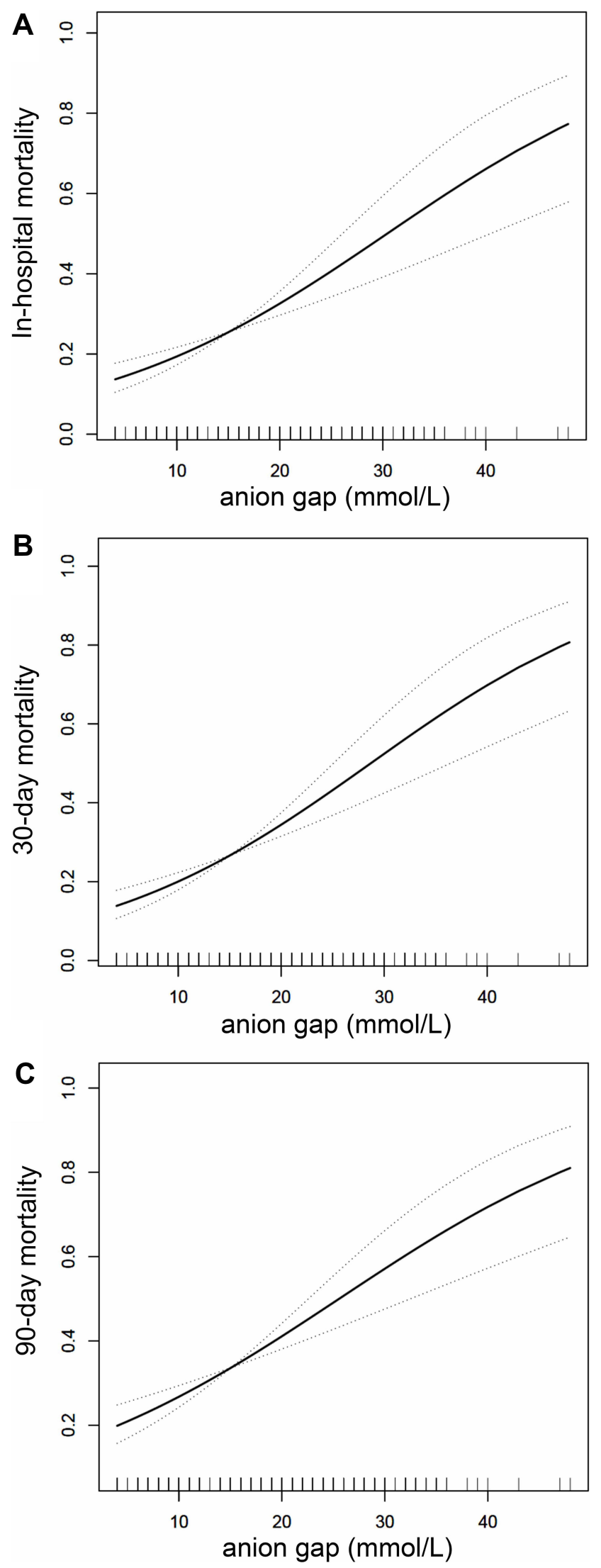

Figure 2 Non-linear relationship between AG and in-hospital mortality (A), 30-day mortality (B), and 90-day mortality (C) of DIC patients.

\section{Discussion}

DIC is a critical illness which always results in short-term mortality. So, we introduced the in-hospital and 30-day mortality as our outcomes. Moreover, we also introduced the 90-day mortality, a middle-long outcome in this paper, which can more comprehensively evaluate the relationship between AG and all-cause mortality in DIC patients. This study demonstrated that in-hospital, 30-day, and 90-day mortality of DIC patients was positively correlated with AG level. This indicates that high AG is an independentdeath risk factor of DIC patients, which means downregulating AG may be of benefit to DIC. This may provide an important theoretical basis for the treatment of DIC.

AG represents the concentration of unmeasured anions in serum, which is calculated by the formula $\left(\mathrm{Na}^{+}+\mathrm{K}^{+}\right)-$ $\left(\mathrm{Cl}^{-}+\mathrm{HCO}^{-}\right) \cdot{ }^{10} \mathrm{AG}$ is an easily available laboratory indicator and is not influenced by acute respiratory changes. Serum AG can find subtle changes in serum electrolytes and the degree of metabolic acidosis and is a more sensitive indicator of metabolic acidosis. ${ }^{11}$ Many serious diseases are most commonly accompanied with metabolic acidosis and an elevated AG level. ${ }^{10,11}$ Several clinical studies have evaluated the association between serum $A G$ and the clinical outcomes in various diseases. ${ }^{21,22}$ Cheng et $\mathrm{al}^{21}$ demonstrated that increased AG values were associated with elevated risk of allcause mortality in critically ill patients with acute kidney injury. Sahu et $\mathrm{al}^{22}$ assessed the relationship between AG and in-hospital outcomes among consecutive acute myocardial infarction patients. They found that the presence of an initial anion gap acidosis was associated with the risk of death (odds ratio $=4.2,95 \%$ confidence interval $=2.3-7.5$, $P<0.001$ ), independent of other data available at the time of admission. ${ }^{22}$ Our study further confirmed the positive relationship between a high AG level and increased allcause mortality in DIC patients.

In order to prove the usefulness of AG for prognosis in DIC patients, we also evaluated the association between the AG and mortality in non-DIC patients. In total, 36,619 patients of the MIMIC-III database were enrolled in the non-DIC cohort. The in-hospital, 30-day, and 90-day allcause mortality were positively correlated with $A G$ in the non-DIC patients also. Detailed data is displayed in Supplementary Table 1. The results were similar to the DIC cohort. There did not exist a more obvious positive relationship between AG and all-cause mortality in DIC patients than in non-DIC patients. This indicated that AG 
Table 3 Subgroup Analysis of the Relationship Between AG and 30-Day Mortality

\begin{tabular}{|c|c|c|c|c|}
\hline Characteristic & Number of Patients & HR (95\% CI) & $P$-value & $P$ for Interaction \\
\hline \multicolumn{5}{|l|}{ Sex } \\
\hline Female & 1,050 & $1.05(1.03-1.08)$ & $<0.0001$ & 0.7543 \\
\hline Male & $\mathrm{I}, 530$ & $1.06(1.04-1.08)$ & $<0.0001$ & \\
\hline \multicolumn{5}{|l|}{ Age (years) } \\
\hline$<60$ & 1,226 & $1.06(1.04-1.09)$ & $<0.0001$ & 0.2294 \\
\hline$\geq 60$ & $\mathrm{I}, 354$ & $1.04(1.02-1.07)$ & 0.0005 & \\
\hline \multicolumn{5}{|l|}{ Care unit } \\
\hline $\mathrm{CCU}$ & 196 & $1.02(0.93-1.11)$ & 0.7084 & 0.4727 \\
\hline CSRU & 476 & $1.14(1.03-1.25)$ & 0.0084 & \\
\hline $\mathrm{MICU}$ & $\mathrm{I}, 268$ & $1.05(1.02-1.07)$ & 0.0004 & \\
\hline SICU & 386 & $1.05(1.00-1.10)$ & 0.0469 & \\
\hline TSICU & 263 & $1.07(1.00-1.13)$ & 0.0505 & \\
\hline \multicolumn{5}{|l|}{ Causes of DIC } \\
\hline Sepsis & $\mathrm{I}, 465$ & $1.04(1.02-1.06)$ & 0.0003 & 0.1526 \\
\hline Cardiovascular diseases & 424 & $1.11(I .02-1.21)$ & 0.0126 & \\
\hline Trauma & 140 & $1.09(1.00-1.19)$ & 0.0582 & \\
\hline Others & 551 & $1.09(1.04-1.15)$ & 0.0004 & \\
\hline \multicolumn{5}{|l|}{ COPD } \\
\hline No & 2,193 & $1.05(1.03-1.07)$ & $<0.0001$ & 0.3802 \\
\hline Yes & 387 & $1.08(1.03-1.13)$ & 0.0028 & \\
\hline \multicolumn{5}{|l|}{ Diabetes } \\
\hline No & 2,091 & $1.05(1.03-1.07)$ & $<0.0001$ & 0.2487 \\
\hline Yes & 561 & I.08 (I.04-I.I2) & 0.0003 & \\
\hline \multicolumn{5}{|l|}{ Renal failure } \\
\hline No & 2,254 & $1.05(1.03-1.07)$ & $<0.0001$ & 0.0310 \\
\hline Yes & 326 & $1.10(1.06,1.14)$ & $<0.0001$ & \\
\hline \multicolumn{5}{|l|}{ CRRT use } \\
\hline No & 2,359 & $1.06(1.03-1.08)$ & $<0.0001$ & 0.7379 \\
\hline Yes & 221 & $1.05(1.01-1.08)$ & 0.0053 & \\
\hline \multicolumn{5}{|l|}{ Vasopressor drugs use } \\
\hline No & 1,229 & $1.05(1.02-1.09)$ & 0.0023 & 0.9986 \\
\hline Yes & $\mathrm{I}, 35 \mathrm{I}$ & $1.05(1.03-1.08)$ & $<0.0001$ & \\
\hline \multicolumn{5}{|l|}{ Mechanical ventilation use } \\
\hline No & 810 & $1.07(1.04-1.11)$ & 0.0001 & 0.2795 \\
\hline Yes & $\mathrm{I}, 770$ & $1.05(1.03-1.07)$ & $<0.0001$ & \\
\hline
\end{tabular}

Abbreviations: CCU, Coronary Care Unit; CSRU, Cardiac Surgery Recovery Unit; MICU, Medical Intensive Care Unit; SICU, Surgical Intensive Care Unit; TSICU, Trauma/ Surgical Intensive Care Unit; DIC, disseminated intravascular coagulation; COPD, chronic obstructive pulmonary disease; CRRT, continuous renal replacement therapy.

is positively related with all-cause mortality of nearly all of the critically ill patients. Further research is needed to clarify this phenomenon.

Subgroup analysis is extremely important for a scientific study. ${ }^{23}$ In the present study, we conducted detailed subgroup analyses using sex, age, care unit, causes of DIC, COPD, diabetes, renal failure, CRRT use, vasopressor drugs use and mechanical ventilation use as stratification variables. The positive association between AG and 30-day mortality was more obvious in DIC patients with renal failure than without renal failure. The possible reason is DIC patients with renal failure are more likely to complicate with metabolic acidosis and higher AG.

It should be noted that, regarding the diagnosis of DIC, there exists three main criteria recommend by different 
associations, including the Scientific and Standardization Committee (SSC)/International Society of Thrombosis and Haemostasis (ISTH), JAAM, and Japanese Ministry Health, Labour and Welfare (JMHLW). ${ }^{24}$ A previous study compared the different diagnostic criteria, and found the JAAM scoring system was the most sensitive one. $^{25}$ So, we introduced the JAAM scoring system in this study. However, despite the JAAM scoring system having a high sensitivity in the diagnosis for DIC patients, its specificity was relatively low and it was useful mainly for sepsis. ${ }^{26}$ So, we have enrolled non-DIC patients in our study.

In 2015, Kury et $\mathrm{al}^{27}$ sought to reproduce, as a computational retrospective study in the MIMIC-II database, a large prospective clinical study. ${ }^{28}$ They identified 2,257 eligible patients in MIMIC-II and found their results remarkably agreed with the original prospective study. This further demonstrated that the results drawn from a retrospective study regarding DIC in the MIMIC database were reliable.

Our work has a number of strengths. First, our studies were the first research series regarding the mortality risk factors of DIC patients. This study focused on AG and was the first to investigate the relationship between $\mathrm{AG}$ and the mortality in DIC patients. Second, this study enrolled 2,654 patients, which is a very large sample size for the clinical study of DIC. Third, we explored both the linear and non-linear relationship. Fourth, we analyzed the exposure variable (AG) as not only a continuous variable but also a categorical variable, and calculated the hazard ratio using binary logistic regression models. Such a method can minimize the incidence of contingency in statistical analysis, and enhance the reliability of the final results.

There are some limitations to our study. First, some variables, including FDP, D-dimer, C-reactive protein, lactate, $\mathrm{pH}$, thrombin, antithrombin, thrombin-antithrombin complex, plasmin-alpha 2-antiplasmin complex, procalcitonin, and the severity of the causes of DIC, may also be related to the prognosis of DIC patients. However, because of a high percentage of missing data or unable obtaining for these variables, we could not include these variables in our data analyses. Second, regarding treatment, we only analyzed CRRT, mechanical ventilation, and vasopressor. The prognosis of DIC patients may also be influenced by other therapeutic measures, such as plasma transfusion, cryoprecipitation transfusion, and platelet transfusion. However, we could not obtain these data from the MIMICIII database. Thirdly, as a single-center retrospective study, selection bias was inevitable; therefore, multicenter registry, prospective studies are needed to confirm this findings.

\section{Conclusion}

Serum AG is positively related with all-cause mortality in DIC patients.

\section{Data Sharing Statement}

The datasets are publicly available in the https://mimic. physionet.org/.

\section{Funding}

This study was supported by grants from the Clinical Medical Technology Innovation Guide Project of Hunan (No. 2017SK51305).

\section{Disclosure}

The authors declare that they have no conflicts of interest for this work.

\section{References}

1. Squizzato A, Hunt BJ, Kinasewitz GT, et al. Supportive management strategies for disseminated intravascular coagulation. An international consensus. Thromb Haemost. 2016;115(5):896-904.

2. Gando S, Levi M, Toh CH. Disseminated intravascular coagulation. Nat Rev Dis Prim. 2016;2:16037.

3. Iba T, Levy JH, Warkentin TE. Diagnosis and management of sepsis-induced coagulopathy and disseminated intravascular coagulation. J Thromb Haemost. 2019;17(11):1989-1994.

4. Costello RA, Nehring SM. Disseminated Intravascular Coagulation. In: StatPearls. Treasure Island (FL): StatPearls Publishing LLC.; 2020.

5. Gando S, Otomo Y. Local hemostasis, immunothrombosis, and systemic disseminated intravascular coagulation in trauma and traumatic shock. Crit Care. 2015;19(1):72.

6. Shirahata A, Shirakawa Y. [New approach to the diagnosis of disseminated intravascular coagulation in childhood]. Nihon Rinsho. 1993;51(1):61-66. [Japanese]

7. Crimi E, Taccone FS, Infante T, Scolletta S, Crudele V, Napoli C. Effects of intracellular acidosis on endothelial function: an overview. J Crit Care. 2012;27(2):108-118.

8. Iba T, Levi M, Levy JH. Sepsis-Induced Coagulopathy and Disseminated Intravascular Coagulation. Semin Thromb Hemost. 2020;46(1):89-95.

9. Kraut JA, Madias NE. Serum anion gap: its uses and limitations in clinical medicine. Clin J Am Soc Nephrol. 2007;2(1):162-174.

10. Jones BJ, Twomey PJ. The anion gap revisited. Int $J$ Clin Pract. 2009;63(10):1409-1412.

11. Brubaker RH, Vashisht R, Meseeha M. High Anion Gap Metabolic Acidosis. In: StatPearls. Treasure Island (FL): StatPearls Publishing LLC.; 2020.

12. Glasmacher SA, Stones W. Anion gap as a prognostic tool for risk stratification in critically ill patients - a systematic review and meta-analysis. BMC Anesthesiol. 2016;16(1):68.

13. Yang SW, Zhou YJ, Zhao YX, et al. The serum anion gap is associated with disease severity and all-cause mortality in coronary artery disease. J Geriatr Cardiol. 2017;14(6):392-400. 
14. Abramowitz MK, Hostetter TH, Melamed ML. The serum anion gap is altered in early kidney disease and associates with mortality. Kidney Int. 2012;82(6):701-709.

15. Chen Q, Chen Q, Li L, et al. Serum anion gap on admission predicts intensive care unit mortality in patients with aortic aneurysm. Exp Ther Med. 2018;16(3):1766-1777.

16. Johnson AE, Pollard TJ, Shen L, et al. MIMIC-III, a freely accessible critical care database. Sci Data. 2016;3:160035.

17. Lambden S, Laterre PF, Levy MM, Francois B. The SOFA score-development, utility and challenges of accurate assessment in clinical trials. Crit Care. 2019;23(1):374.

18. Jacome T, Tatum D. Systemic Inflammatory Response Syndrome (SIRS) score independently predicts poor outcome in isolated traumatic brain injury. Neurocrit Care. 2018;28(1):110-116.

19. Capuzzo M, Moreno RP, Le Gall JR. Outcome prediction in critical care: the simplified acute physiology score models. Curr Opin Crit Care. 2008;14(5):485-490.

20. Gando S, Iba T, Eguchi Y, et al. A multicenter, prospective validation of disseminated intravascular coagulation diagnostic criteria for critically ill patients: comparing current criteria. Crit Care Med. 2006;34(3):625-631.

21. Cheng B, Li D, Gong Y. Serum anion gap predicts all-cause mortality in critically ill patients with acute kidney injury: analysis of the mimic-iii database. Dis Mark. 2020;2020:6501272.
22. Sahu A, Cooper HA, Panza JA. The initial anion gap is a predictor of mortality in acute myocardial infarction. Coron Artery Dis. 2006;17 (5):409-412.

23. Vandenbroucke JP, von Elm E, Altman DG, et al. Strengthening the Reporting of Observational Studies in Epidemiology (STROBE): explanation and elaboration. PLoS Med. 2007;4(10):e297.

24. Wada H, Matsumoto T, Yamashita Y. Diagnosis and treatment of disseminated intravascular coagulation (DIC) according to four DIC guidelines. J Intesive Care. 2014;2(1):15.

25. Kury FS, Cimino JJ. Computationally comparing and analyzing all published scoring systems for diagnosis of disseminated intravascular coagulation. Stud Health Technol Inform. 2015;216:956.

26. Gando S, Wada H, Asakura H, et al. Evaluation of new Japanese diagnostic criteria for disseminated intravascular coagulation in critically ill patients. Clin Appl Thromb Hemost. 2005;11(1):71-76.

27. Kury FS, Huser V, Cimino JJ. Reproducing a prospective clinical study as a computational retrospective study in MIMIC-II. AMIA Annu Symp Proc. 2015;2015:804-813.

28. Gando S, Saitoh D, Ogura H, et al. A multicenter, prospective validation study of the Japanese Association for Acute Medicine disseminated intravascular coagulation scoring system in patients with severe sepsis. Crit Care. 2013;17(3):R111.
International Journal of General Medicine

\section{Publish your work in this journal}

The International Journal of General Medicine is an international, peer-reviewed open-access journal that focuses on general and internal medicine, pathogenesis, epidemiology, diagnosis, monitoring and treatment protocols. The journal is characterized by the rapid reporting of reviews, original research and clinical studies

\section{Dovepress}

across all disease areas. The manuscript management system is completely online and includes a very quick and fair peer-review system, which is all easy to use. Visit http://www.dovepress.com testimonials.php to read real quotes from published authors. 